\title{
Design and Realization of a Condition Management System for the Gateway Electrical Energy Metering Device
}

\author{
Chao Tang ${ }^{1}, \mathrm{Jiao}^{2 i^{2, *}}$, Bifeng Xiong ${ }^{1}$, Jufang $\mathrm{Xie}^{1}$ and Dong $\mathrm{Hu}^{1}$ \\ ${ }^{1}$ College of Engineering and Technology, Southwest University, Chongqing, 400715 - China \\ ${ }^{2}$ University Library, Southwest University, Chongqing, 400715 - China
}

Received 15 October 2013 ; Accepted 10 December 2013

\begin{abstract}
With the construction of firm and intelligent power grid in China, it is difficult for the traditional management method of electrical energy metering device to meet the prospecting requirements. Using the computer and internet techniques to realize the information and intelligentization of the electrical energy metering management has become a necessary guarantee of improving power supply ability, marketing control, and customer service. This paper introduced a kind of large and intelligent condition management system of the gateway electrical energy metering device. The key technologies and realize process were analyzed. Moreover, a detailed description of the application modules such as the GIS smart display of metering point, the condition management of metering devices and the visual monitoring of metering point was presented. The trial operation in the selected transformer substations and the power stations of Chongqing Power Electrical Corp. indicated that, the condition management system is very open, safety and efficient. According to the data exchange with the production and scheduling platform, the system improved the efficient operation of the electrical energy metering devices. Meanwhile, combined with the real-time visual monitoring, the condition management system improved the prevention ability of electricity filching, realized the unified automatic large-scale management of electrical energy metering devices.
\end{abstract}

Keywords: Electrical Energy Metering, On-line Monitoring, Fuzzy Comprehensive Evaluation, DRBAC Method, Fuzzy Expert System

\section{Introduction}

In order to strengthen the electric power measuring management, the computer information management is introduced to many domestic electric power companies. In China, Shanghai Power Meter Plant had developed asset management system of electronic measuring since 1996 and it had been put into use by the end of 1997 . However, the electronic measuring management system didn't serve for the power enterprise as an independent management information system in the early stage, but served in the information system such as information system of power marketing management and technical support system of power marketing. Therefore, disadvantages of such systems can be concluded that they are of single function, weak comprehensive application and lack of workflow driving mechanism.

In recent years, a lot of reformation and innovation work has been done by domestic technical personnel based on the monitoring and management of the electric power measuring device. The literature [1] elaborated the necessity of application of remote test and monitoring system on the electric measuring device and system principle. The test run demonstrates that the application of system can ensure safe,

\footnotetext{
*E-mail address: $41847130 @ q q . c o m$

ISSN: 1791-2377 (C) 2013 Kavala Institute of Technology. All rights reserved.
}

reliable and real-time monitoring of the electronic power measuring device. The literature [2] showed a new work mode: to get electric energy data by remote acquisition, realtime transmitted to the laboratory for comparison and analysis, and the problem can be solved in time by personnel. The literature [3] and [4] demonstrated automobile detection system to the electric power measuring device, which has power meter tested with a method of 'one choice from the multiple-way' to the electric energy metering devices through a branch one. The literature [5] presented an on-line monitoring method to the measurement device based on the electric information acquisition system. However, there are strict step limitations when using this method and it cannot be tested in project application. The literature [6] showed a kind of electric power measurement on-line monitoring system. Through application of the system, the measurement management personnel can find the fault of measurement device in time and inform others to deal with it. Also, the system can record the load curve of fault. When the error of measurement device is out of limitation, it is easier for the measurement management personnel to find the fault and take measures to deal with it in time. However, currently there are disadvantages in the application of electric power measurement monitoring system and the monitoring capacity of the single equipment is small [7-9].

At the same time, through the construction for many years, Electric Power Research Institute of Chongqing 
Chao Tang, Jiao Li, Bifeng Xiong, Jufang Xie and Dong Hu

\section{/Journal of Engineering Science and Technology Review 6 (3) (2013) 56 - 61}

Power Electrical Corp had basically reached the requirement of automatic management. Such as automatic logistics system, automatic verification and seal technology, and a series of national laboratory had been built, which formed a systemic and complete precise management to the electric power meter. However, there were problems such as inadequacy of the status management to the on-site meter especially the gateway meters device, lack of efficient monitoring and management to the on-site electric stealing.

Considering all the issues above, Electric Power Research Institute of Chongqing Power Electrical Corp. gets organized to develop a set of gateway electric power meters device status management system, which used Windows Server 2008 as its operational system platform, Oracle database management system and VS .NET system as its development tools, adopted $\mathrm{n}$ tiers system architecture and reusable component technique to realize automation, intelligent online monitoring and maintenance. The trial operation tests of the system were performed in more than $20220 \mathrm{kV}$ substations in Chongqing, the result showed that the system combines with the front-end hardware to form an multifunctional and multi-purpose electric power metering analysis research platform, which had the function of electric power meters and test, data analysis and data collecting, chart drawing and so on of high capacity and can be extended for database support.

\section{Architecture of the system}

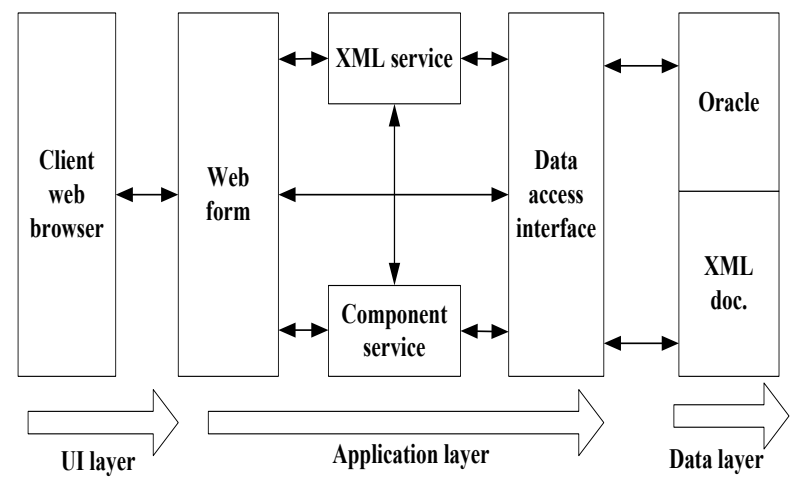

Fig. 1. System Structure Diagram

This system adopts $\mathrm{b} / \mathrm{s}$ architecture. The structure diagram of the system is as shown in Figure 1.

The system adopts ASP. NET to realize b/s architecture, to operate on access scheme of the electric power system ORACLE database and the client resources, which is used for management on the status of metering device, the operation of the metering device and GPS information.

In addition to such advantages that ASP .NET can run faster than the traditional ASP, has server controls, and is easier for writing code in adopting 'code-behind' method, the advantage of the ASP .NET is that it is available for the real-time update of application program, it is available for dynamic compilation files when necessary and can save the compilation result for reusing the subsequent requires. ASP .NET can also automatically detect errors (such as memory leaks, deadlock) and renew them to make certain of the availability of application program. For example, when detecting the memory leaks, ASP .NET will automatically start a new ASP .NET working process copy, and will position all the new requirements to the process. When the old process asks for hang, it will be properly dealt with and release the leaked memory.

Secondly, when the system architecture design has been completed, it should have information system risk evaluated in the safety analysis method. The safety risk evaluation of information system is an evaluation process to the safety properties such as information confidentiality, integrity and availability of information system and information to be dealt with, transferred and stored.

The risk of information system is related to such three elements as asset, threat and fragility. The risk influence of these factors to the information system is uncertain and of ambiguity. The security risk evaluation of information system based on the fuzzy comprehensive evaluation theory is to assign each threat risk an index value based on the expert experience, to calculate in fuzzy evaluation matrix and to make an overall evaluation to the variety of risk factors that have a threat to the information system.

Finally, an expert system is formed based on the experience of the famous experts both at home and abroad and the experience of Chongqing Power Electrical Corp after building the safe information system. Also, a set of status evaluation mechanism of large-scale remote electric power metering device has been formed based on the design of fuzzy method.

\section{Crucial technique and realization of the system}

\subsection{Optimization technique of system programming} 3.1.1 Optimization of system performance

This system makes use of buffering technique and selecting buffering time is the crux of performance optimization. As personnel in power department does not frequently update database, data buffering model of ASP.NET is selected to design system page. Data which is usually stored or accessed or relatively important data shall be stored in internal storage so that response time of system is reduced. Buffering time is set to be a proper time and what kind of data on the page are to be buffered as well as when to empty buffering and renew write-in for inquiry are designated. This method enhances performance of the system and brings convenience for working personnel.

\subsubsection{Code optimization system}

Code quality directly influences performance and maintainability of system. Therefore, good programming thought can greatly improve performance and maintainability of system. Some methods are as follows:

(1) Reduce connection with database as much as possible. It will take a lot of time to repeatedly connect database. With increase of data amount and rapid increase of internal storage consumption, resources of connection pool will not be released in a short time and finally, the system will break down.

(2) Making use of variable distinct. If statement data repeatedly emerge at the process of system development, variable distinct can be used to solve all problems. Codes are as follows: SELECT distinct Level Type FROM WHERE.

\subsection{Risk evaluation of information system}

\subsubsection{Threat confronted by power information system}

According to different resources of threats, main safety threats confronted by system can be divided to the following four categories.

(1) Safety risk which is brought by safety fragility and defect of information system. Safety fragility and defect of 
Chao Tang, Jiao Li, Bifeng Xiong, Jufang Xie and Dong Hu

/Journal of Engineering Science and Technology Review 6 (3) (2013) 56 - 61

information system come from physical safety defects of hardware which result from information system, design loopholes of software assembly (including operation system, application platforms and application business, function redundancy, logistical confusion and safety dangers from complicity, reliability and controllability of network communication protocol.

(2) Safety threats from the internal. The majority of malicious attack is from the internal and reasons are numerous and complex, such as negligence of duty of system administer, fault of operation staff, malicious attack of internal resentful personnel, etc. There are a lot of malicious attacks, such as attempting to make resources reject service, eavesdropping, stealing information, pretending as legal users or system process, altering information content, making use of support system disadvantages, malicious codes, forging legal system service, malicious secret leakage, hacker technique, virus, etc. But their objectives are to steal secrets and damage.

(3) Safety threats from exterior environment. Regardless of network composition ways in the corporate, safety threats to networks in the corporate of Internet network always exist. Hackers, espionages or other criminals can adopts methods such as eavesdropping by building wires, network monitoring and network scanning to intercept information, which has certain safety dangers and needs to be prevented. Furthermore, in terms of management, contact and using of terminal equipment in power information system by external personnel shall be eliminated.

(4) Safety threats from nature. It includes transient stop of system or service stop which may be caused by natural disaster such as flood, typhoon and earthquake.

\subsubsection{Risk evaluation model}

(1) Confirmation of safety risk factor set of system

Suppose $S$ is all safety risk factor set of information system and put factors of similar characters in the same group. Suppose factors in $S$ are put in one group, that is,

$$
S=\left\{S_{1}, S_{2}, \ldots, S_{L}\right\}
$$

Where $S_{i}$ represents factor in $i$ group in $S$, $i=1,2, \ldots, L$, Since each $S_{i}$ has $n$ factor risk sets, it can be expressed as $S_{i}=\left\{S_{i 1}, S_{i 2}, \ldots, S_{i n}\right\}$. Therefore, safety risk factor sets are divided into multi-layer sets.

(2) Definition of safety risk index

Safety risk index $V$ represents influence degree of consequence of safety risk of information system to information system.

$$
V=\left\{V_{1}, V_{2}, \ldots, V_{m}\right\}
$$

Where $m$ represents quantity of risk index sets and $V_{j}$ represents safety risk index $j=1,2, \ldots, m$.

(3) Confirmation of weight coefficient of safety risk factors

Weight of all factors in $S_{i}$ versus safety risk index set

$V$. Coefficient is expressed with matrix as follows:

$$
A_{i}=\left\{A_{i 1}, A_{i 2}, \ldots, A_{i n}\right\}
$$

Where sum of all coefficients are 1 .

(4) Calculation of comprehensive evaluation matrix of safety risk

Firstly confirm single factor $S_{i n}$ versus fuzzy evaluation matrix $R_{i}$ of single factor of risk index set $V$ according to fuzzy deduction methods.

Then confirm fuzzy comprehensive evaluation matrix $B$ of safety risks of high-level factor $S$.

Finally comprehensively calculate safety risk $S$ of various safety risk factors.

\subsubsection{Safety risk analysis and preventive measures of the system}

The system involves several links. With the methods above, it can be concluded that remote control part has the biggest safety danger of system.

Power system automation demands realization of remote control of substation. Currently the critical technique which influences remote control is ensuring safe visiting and control for substation equipment. To prevent illegal visiting, power exceeding visiting and unsuitable operation of substation equipment from damaging safe operation of substation according to required methods and confirm normal operation of substation, remote control of substation must have good visiting control mechanism.

Role Based Access Control Model which is widely applied in power information system currently separates users from authorities by introducing 'roles'. But for large scale system in power industry, numerous users and wide resources make user-role assignment of complex roles (roles which are confirmed by multiple attributes according to certain rules) a heavy task. Therefore problems come into being. First is reduction of efficiency and second is complexity aggregation of role definition as a result of interoperation which exists between different areas.

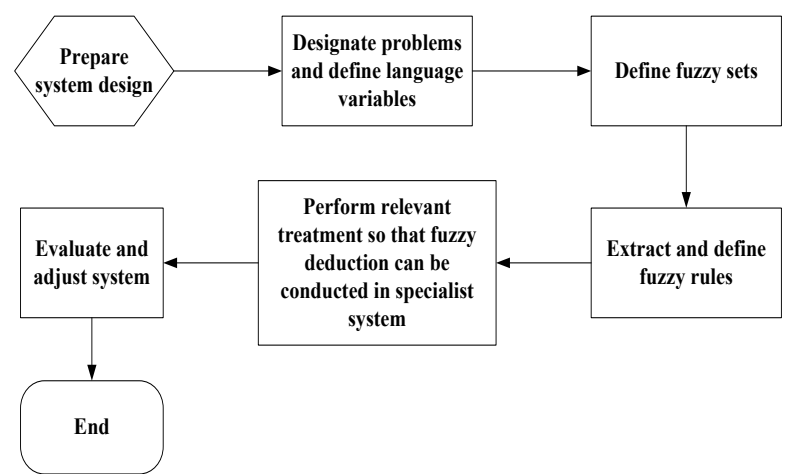

Fig. 2. MD_ABAC Algorithm

According to literature, our system adopts MD-ABAC decision methods to realize visiting module.

Attribute Based Access Control Model in multi-domain environment MD_ABAC $=\{S, R, E, A, P, A A, P A, M A, M P\}$, of which $\mathrm{S}, \mathrm{R}, \mathrm{E}, \overline{\mathrm{A}}, \mathrm{P}, \mathrm{AA}, \mathrm{PA}, \mathrm{MA}$ and $\mathrm{MP}$ respectively represent subject, resources, environment, attribute, power, attribute assignment, power assignment, meta-attribute and meta-policy.

The flow diagram is showed in Fig. 2. 
Chao Tang, Jiao Li, Bifeng Xiong, Jufang Xie and Dong Hu

\section{/Journal of Engineering Science and Technology Review 6 (3) (2013) 56 - 61}

\subsubsection{Status evaluation of measuring device based on} fuzzy specialist system

In light of domestic problems that gate measuring device lacks efficient status management and on-site electricity stealing cannot be effectively supervised, method of fuzzy status evaluating for measurable operation data based on onsite measuring device is put forward with technical experience for many years of Chongqing Power Electrical Corp and intelligence of domestic famous specialists, scholars and information system researchers. With evaluation matrix, the method performs pertinence evaluation for real time operation status of measuring device and it comes true in this system. Basic design process is showed in Fig. 3.

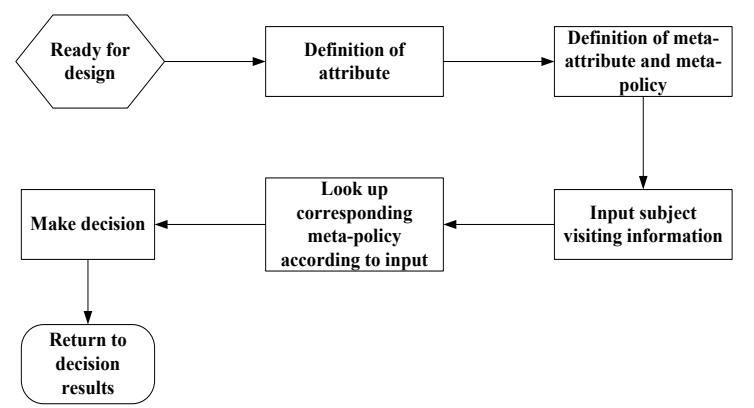

\section{Main functions of system and on-site operation}

The set of 'status management system of gate electric energy measuring device' regards entire life cycle of gate measuring device as timer shaft and covers information records before operation of measuring device and operation status monitoring after operation of measuring device. Corresponding diagrams shall be built according to data of operation status after operation (including normal data and abnormal data). Analysis of various diagrams can be used to judge heath status of current measuring device so that it can provide decision evidence for decision level, operating management personnel and business analysis personnel. Furthermore, the system can perform efficient data interchange with production dispatching platform of measuring center to realize higher application of effective operation of electric energy measuring device.

The system is featured by beautiful login interface, strong humanization and easy for operation. Its main login interface is shown in Fig. 4.

Fig. 3. Design flow diagram of fuzzy expert system

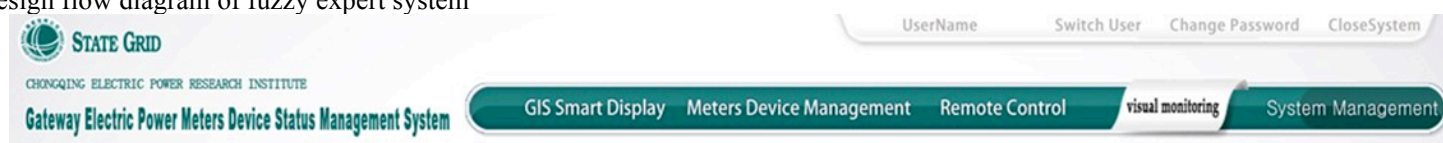

Fig. 4. System log-in interface

\subsection{Function of GIS smart display}

The function can realize management of Chongqing power grid topological graph, freely increase and delete power grid joints, automatically traverse all gate sheets which are inserted in terminals and intelligently acquire connection information and perform power grid map edition, etc.

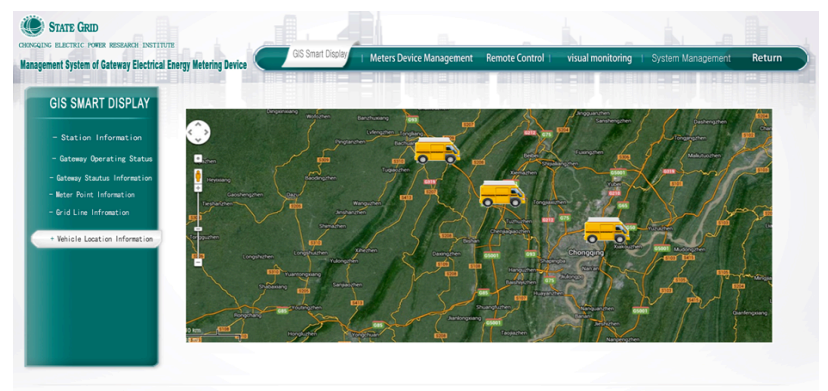

(6) Strar Gro

Fig. 5. The interface of the GIS smart display
Regarding relevant operation information of remote measuring points as principal lines and selectively display remote measuring points with geographic position and type of measuring point, voltage classes, unit property, property of electric quantity, the function can dynamically show selected factory station on the map of Chongqing (Fig. 5).

Furthermore, the system has connection of vehiclemounted GPS position system with Chongqing Power Electrical Corp, which can realize accuracy location of working vehicles and reach the objective of prompt dispatching and rapid emergency maintenance.

Relevant technical management personnel can operate all gate points through management system. When one gate is selected, status information of primary wiring of the gate can selectively display and operation of offside verification point can display too. The latest information of electricity can dynamically display in corresponding gates and operation of gates shall be marked with different colors. Furthermore, chaining can be provided to respectively visit factory station 
Chao Tang, Jiao Li, Bifeng Xiong, Jufang Xie and Dong Hu

\section{/Journal of Engineering Science and Technology Review 6 (3) (2013) 56 - 61}

information of gate, remote measuring point information, asset information collection information, load condition, event information, verification data and formula of electric quantity, etc.

\subsection{Status management of measuring device}

4.2.1 Management of entire life cycle of measuring device Unified management of design scheme review of gate measuring device, equipment installation, completion acceptance, on-site inspection, rotation, fault treatment, change, dismantling, configuration information, asset information of measuring device, information of collection equipment, deviation testing information of measuring device, information of real time electric energy, information of primary wiring of remote measuring point, locating information, alarm information of failure exception and variation trend information of deviation influenced by environment can be realized.

It can perform information smart display of real time power information and electricity, verification information and variation trend information of deviation influenced by environment, conduct fault treatment of remote measuring point and comprehensive analysis, evaluate status characteristics of measuring device of electric energy and give reference decision opinions.

It can immediately arrange work recovery for fault points and adjust cycle re-testing period according to operation status; it can realize controlled management on life cycle of confirmation for measuring scheme of electric energy measuring device on gate, selection of measuring instrument, ordering acceptance, re-testing, overhaul, safeguard, acceptance of installation and completion, operation maintenance, on-site inspection, periodical retesting (rotation), spot check, fault treatment and discarding.

\subsubsection{Real time on-line monitoring of operation status of measuring device}

Function of this part can realize monitoring and verification of deviation date of on-site and remote measuring device.

Control terminal shall perform data reading of electric energy meter and recall collection data according to defined cycles or instant response. It can also read operation data of gate electric energy meter such as charging data, instantaneous value, load curve, event records, etc.

It can analyze deviation trend of metering device: It can calculate deviation electricity of remote measuring points with electricity data of remote measuring point (formula) according to calculation results of comprehensive deviation of each remote measuring point. Based on this, mathematical modules can be built and estimation of weighting annual deviation electricity can be performed with relevant interpolation algorithm.

It can integrate dynamic real time information, perform electricity balance monitoring of net remote measuring point and offside reference point, promptly find operation abnormalities of electricity measuring device and provide reference evidence for fault treatment and comprehensive analysis of remote measuring point.

It can provide complete warning function: Warning of abnormal status of measuring device, warning of deviation status out of tolerance, inability to read relevant electricity data and warning of other functional faults. All warning functions and threshold values can be freely set.
4.3 Management of visual monitoring of the remote measuring point

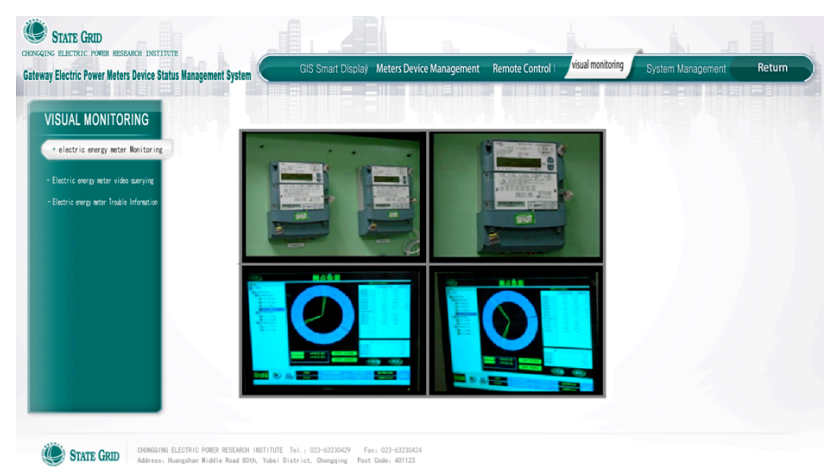

Fig. 6. The interface of visual monitoring of the remote measuring point

This part can provide instant image recognition and judge whether display of electric energy meter is normal if it is combined with on-site visual collection units of remote measuring points; it can monitor image recognition of operation environment change to reach the function of preventing electricity stealing; it supports image record and access to USB device and GPS timing system (Fig. 6).

\subsection{On-site application}

Electricity Measuring Center of Chongqing Power Electrical Corp. serves 12 Administrations of Power Supply and 25 municipal Power Supply Companies of 40 municipalities of Chongqing with an area of 82,400 square kilometers. Asset management of meter calculation is difficult and the farthest round-trip is nearly 1,400 kilometers.

Operation results of the system in over $20220 \mathrm{kV}$ pilot substations under the direction of Chongqing Power Electrical Corp. prove that the system can perform efficient data interchange with production dispatching platform of measuring meter and realize advanced application of efficient operation of electricity measuring device. Meanwhile, monitoring measures of real time visual images are adopted to greatly improve anti-electricity stealing capacities of electricity measuring device and realize unified and automatic on-line management of mass electricity measuring devices, which is a bran-new automatic management modes of electricity measuring devices. Since it was put into operation, a set of intellectualization, intensification and scientific management system of gate electricity measuring has been basically formed, quantities and workload of re-testing personnel greatly decrease and verification amount of measuring arbitration application by customer decreases by nearly $30 \%$.

\section{Conclusions}

With the construction of strong smart grid in China, using computer technology and network technique to realize information and intellectualization of electricity measuring management becomes a necessary guarantee of improving power supply quality.

A kind of large 'Management System of Electricity Measuring Device' has been developed, with the n-level system structures and adopted and reusable component technique to realize on-line monitoring and maintenance of automation and intellectualization of electricity measuring device.

Operation results of the system in over $20220 \mathrm{kV}$ pilot substations under the direction of Chongqing Power 
Chao Tang, Jiao Li, Bifeng Xiong, Jufang Xie and Dong Hu

/Journal of Engineering Science and Technology Review 6 (3) (2013) 56 - 61

Electrical Corp. proved that the system forms multifunctional and multi-purpose analytical and research platform of electricity measuring for electricity measuring test, analysis, data summarization and chart drawing. The system can perform efficient data interchange with production dispatching platform of measuring meter and realize advanced application of efficient operation of electricity measuring device.

In the meantime, monitoring measures of real time visual images are adopted to greatly improve anti-electricity stealing capacities of electricity measuring device and realize unified and automatic on-line management of mass electricity measuring devices, which is a brand-new automatic management mode of electricity measuring devices.

\section{Acknowledgements}

The authors wish to thank the National Natural Science Foundation of China (Project for Young Scientists Fund, Grant No. 51107103) and the Natural Science Foundation Project of CQ CSTC (Grant No. cstcjjA90013) for the financial support they provided.

\section{References}

1. Yang Jun; Jia Nan; Qi Yan-shou, “Application of Electric Metering Device Remote Verification Monitoring System in Qinghai Power Grid”, Qinghai Electric Power, 2011, Volume:32, Issue:2, pp.28-32

2. Xia Min, "The Research of the Remote Energy Metering Devices Detection Analysis Management System”, Electrical Engineering magazine, 2011, Volume:30, Issue:7, pp.66-81

3. Kong De-shi; Chen Lei; He Bing; Wang Lin, "Function upgrading and application of Chengdu city electrical energy remote acquisition system", Power Demand Side Management, 2011, Volume:31, Issue:4, pp.54-56

4. Chen Zhuo-ya; Qin Nan; Zhou Fu-hai; Pan Hui; Feng Lei, "Design of remote on- line detecting system for power metering device", Electrical Measurement \& Instrumentation, 2008, Volume:45, Issue:510, pp.27-30

5. Hu Zhan-ting; Qin Nan; Sun De-quan, "Online monitoring based on the information collection of power metering device", Journal of Beijing Dianli Gaodeng Zhuanke Xuexiao Xuebao, 2012, Volume:29, Issue:3, pp.3-4
6. Li Jian, "The trial example of Online monitoring system of power metering device", Sichuan Electric Power Technology, 2011, Volume:35, Issue:1, pp.34-38

7. Sorabh Gupta; P.C. Tewari, "Simulation Modelling In a Availability Thermal Power Plant", Journal of Engineering Science and Technology Review, 2011, Volume:4, Issue:2, pp.110-117

8. Sadeghkhani I.; Ketabi A.; Feuillet R., "Artificial Neural Network Based Method to Mitigate Temporary Overvoltages", Journal of Engineering Science and Technology Review, 2011, Volume:4, Issue:2, pp.193-200

9. Peng Du; Huimin Li; Youping Fan, "Application of Impulse Control in Smart Grid Price System", Journal of Engineering Science and Technology Review, 2013, Volume:6, Issue:2, pp.5761 